\title{
NUESTROS COLABORADORES
}

\section{SABERES Y RAZONES}

Marisol Pérez Lizaur es desde 1997 docente del posgrado en antropología social de la Universidad Iberoamericana, donde estudió la licenciatura, la maestría y el doctorado. Fue coordinadora de ese programa y es miembro del Sistema Nacional de Investigadores, nivel II. Trabajó como asesora en la Dirección de Coordinación Educativa y en la Subsecretaría de Investigación y Educación Tecnológica de la Secretaría de Educación Pública. Fue investigadora en la Dirección General de Desarrollo Tecnológico de la Universidad Nacional Autónoma de México. Ha impartido clases la Escuela Nacional de Antropología e Historia y ha fungido como directora de tesis de posgrado en varios programas y universidades. Sus temas de investigación son las universidades como organizaciones, el parentesco urbano, las empresas familiares, las organizaciones, la tecnología y las elites.

Roger Magazine es doctor en antropología social por la Universidad de Johns Hopkins en Baltimore, Maryland, Estados Unidos. Desde 1999 es profesor e investigador del Programa de Posgrado en Antropología Social de la Universidad Iberoamericana y es miembro del Sistema Nacional de Investigadores, nivel II. También forma parte de la Academia Mexicana de las Ciencias desde 2013. Es autor de varios libros, artículos y capítulos de libros sobre aficionados al futbol, niños de la calle en la ciudad de México, nociones y prácticas locales de la persona, la acción y la alteridad en el México rural.

Laura González trabaja en la Indiana University of Pennsylvania como investigadora asociada y dirige el trabajo de campo sobre poblaciones binacionales en Uriangato, Guanajuato, México y en la Kennett Square, Pensilvania, Estados Unidos, en torno a la salud de inmigrantes mexicanas mujeres y niñas. Ha publicado numerosos artículos e informes en México y Estados Unidos sobre la migración y el impacto en las familias, sociedades, economías, culturas y políticas. Ha sido reconocida por su labor de investigación con el $29^{\text {th }}$ Distinguished University Faculty Award de la Texas Association of Chicanos in Higher Education (2004), el Women of Spirit Award del American Jewish Congress Southwest, Dallas Region (2005), el Profiles in Leadership Award (2008), el Women of Distinction Award de la American Association University Women (2008), y en 2012 participó en la Cátedra "Ángel Palerm". Forma parte de consejos y es consultora de organizaciones como consulados mexicanos, centros comunitarios hispanos y latinos, Casa Guanajuato of Dallas, Texas, Casa Guanajuato of Kennett Square y La Comunidad Hispana in Kennett Square, entre otras.

Víctor García es profesor distinguido 2013-2104 y director del Mid-Atlantic Addiction Research Institute de la Indiana University of Pennsylvania. Es también profesor de antropología e imparte cursos sobre latinos, América Latina, sus diásporas y métodos de trabajo de campo en etnografía. Ha sido objeto de distinciones por su trabajo de investigación acerca de las poblaciones de granjeros y el uso de sustancias entre trabajadores inmigrantes. Es miembro del National Hispanic Science Network on Drug Use. Ha presentado su trabajo en artículos, capítulos de libros, informes de investigación, documentos de trabajo, además de conferencias nacionales e internacionales. Parte de su investigación se concentra en aspectos de la educación de la población latina y la necesidad de reclutarla y retenerla en estudios superiores. Este trabajo se ha publicado en un libro en coautoría y en revistas académicas, como el Hispanic Journal of Higher Education.

Carlos Giménez Romero es catedrático de antropología social y aplicada en la Universidad Autónoma de Madrid (UAM) y director del Instituto de Migraciones, Etnicidad y Desarrollo Social (Imedes). En su tesis doctoral (1985), dirigida por Carmen Viqueira, examinó el régimen comunal agrario de España y México, 
con trabajo de campo en el Valle de Toluca, Estado de México. Sus temas de investigación son campesinado y sociedades de regadío; migraciones internacionales, codesarrollo y relaciones interculturales; cambio social, y conflicto y mediación. Combina teoría y práctica, impulsa numerosos proyectos de antropología implicada y de orientación pública. En la actualidad, es delegado del rector para la coordinación de los institutos de investigación de la UAM y coordina el doctorado en el Departamento de Antropología. Es director científico de un macro proyecto teórico-aplicado de intervención comunitaria intercultural en una amplia red de barrios de distintas localidades españolas.

Gustavo Lins Ribeiro es profesor titular de la Universidad de Brasilia. Es presidente de la Associação Nacional de Pós-Graduação e Pesquisa em Ciências Sociais de Brasil y primer presidente del World Council of Anthropological Associations. Pertenece al Consejo Nacional de Desarrollo Científico y Tecnológico Investigador, nivel 1A, de Brasil. Ha escrito y compilado 20 libros en portugués, español e inglés, ha publicado más de 170 artículos y capítulos de libros en seis lenguas en 21 países sobre globalización, transnacionalismo, cibercultura, desarrollo y antropologías mundiales. Ha formado parte de 18 consejos editoriales de revistas como Alteridades (México), American Anthropologist, American Ethnologist y Current Anthropology. Su último libro compilado con Gordon Mathews y Carlos Alba es Globalization from Below. The World's Other Economy (Routledge, 2011).

Robert V. Kemper estudió ciencias sociales e historia en la University of California at Riverside. Maestro y doctor en antropología, desarrolló con su mentor George M. Foster una sólida relación de trabajo con la comunidad de Tzintzuntzan, Michoacán. Inició su carrera académica en la Southern Methodist University en 1972. Fue reconocido como un pionero en el campo de la antropología urbana por su interés en los modos de vida desplegados en las ciudades, en particular en la capital de México y en Dallas. Concentró su estudio en la interacción de la gente cuando se desplaza de las grandes ciudades a comunidades más pequeñas. Los datos que obtuvo en su investigación de largo alcance entre las personas originarias de Tzintzuntzan asentadas en la ciudad de México aportó las bases para comprender el impacto de la migración en el desarrollo de esa comunidad durante los últimos 100 años. Escribió y editó diez libros y nueve números especiales de revistas académicas, publicó 64 artículos, 62 capítulos de libros y numerosas reseñas de libros.

\section{ESQUINAS}

Jaime Guiamet es licenciado en antropología por la Facultad de Humanidades y Artes de la Universidad Nacional de Rosario y docente de la cátedra de economía política en la misma casa de estudios desde 2011. En la actualidad cursa el doctorado en humanidades con mención en antropología mediante el subsidio de una beca de posgrado otorgada por el Consejo Nacional de Investigaciones Científicas y Técnicas. Pertenece al Núcleo de Estudios del Trabajo y la Conflictividad Social, que reúne a investigadores interesados por los problemas del mundo del trabajo. Ha publicado diversos artículos y ponencias en torno a la investigación que lleva a cabo para su tesis doctoral, la cual se concentra en el estudio de las experiencias laborales de trabajadores jóvenes en una cadena multinacional de supermercados en la ciudad de Rosario.

Jaime Torres Guillén es licenciado en letras por la Universidad de Guadalajara. Es maestro en filosofía con especialización en ciencias sociales por el Instituto Tecnológico de Estudios Superiores de Occidente. Es doctor en ciencias sociales con especialidad en antropología social por el Centro de Investigaciones y Estudios 
Superiores en Antropología Social. Se desempeña como profesor del Instituto de Filosofía, A. C. y es director de la revista Piezas en Diálogo, Filosofía y Ciencias Humanas.

Leticia Robles Silva es doctora en ciencias sociales e investigadora de la Universidad de Guadalajara. Su línea de investigación es sobre familia y cuidado. Sus publicaciones se centran en los aspectos culturales del cuidado.

María Daniela Rosas García es estudiante del doctorado en ciencias de la salud pública y se ha desempeñado como asistente de investigación de proyectos sobre cuidado y vejez. Su interés se centra en temas como cuidado y enfermedad crónica.

Juan Antonio del Monte Madrigal es maestro en estudios culturales por El Colegio de la Frontera Norte (Colef), donde recibió la mención honorífica única. Es licenciado en ciencias de la cultura por la Universidad del Claustro de Sor Juana. Ha cursado estudios en sociología en la Facultad de Ciencias Políticas y Sociales de la Universidad Nacional Autónoma de México y ha participado en congresos en torno a la cultura urbana, la juventud y las fronteras. Actualmente coordina la Secretaría Técnica del Consejo Mexicano de Ciencias Sociales con sede en el Colef y es profesor de asignatura en la Facultad de Humanidades y Ciencias Sociales en la Universidad Autónoma de Baja California.

\section{LEGADOS}

Ximena González Munizaga es bibliotecaria desde 1983 y a partir de 1990 se desempeña como subdirectora de las bibliotecas del Centro de Investigaciones y Estudios Superiores en Antropología Social. En 2012 se hizo cargo de la organización de los fondos archivísticos recibidos en donación por la Biblioteca "Ángel Palerm", de la unidad Distrito Federal del Centro y de realizar la propuesta para la formación del archivo histórico de la institución. Es psicóloga social por la Universidad Autónoma Metropolitana-Xochimilco.

\section{RESEÑAS}

Joan J. Pujadas es catedrático en antropología social en la Universidad Rovira i Virgili (URv) de Tarragona, España. En 1977 se doctoró en la Universidad de Barcelona y contribuyó a la creación del núcleo de Antropología Social de la Universidad en Tarragona. Ha sido decano de la Facultad de Letras de la URv (1994-1997) y presidente de la Federación Española de Asociaciones de Antropología (1990-1993 y 1993-1996). Sus campos de especialización son la antropología urbana (migraciones y procesos de urbanización, movilidad y metropolización), la etnicidad (asociacionismo migrante, conflictos urbanos de naturaleza étnica, procesos de hibridación cultural) y cuestiones de epistemología y método etnográfico. Es promotor del acuerdo interuniversitario que en España agrupa a los discípulos y seguidores de la obra de Ángel Palerm.

Hernán Salas Quintanal es investigador titular en el Instituto de Investigaciones Antropológicas de la Universidad Nacional Autónoma de México (unAM). Es doctor en antropología, miembro de la Academia Mexicana de Ciencias, de la Asociación Mexicana de Estudios Rurales y del Sistema Nacional de Investigadores, nivel II. En 2012 recibió el Premio Fray Bernardino de Sahagún por la mejor investigación en el área 
de antropología social que otorga el Instituto Nacional de Antropología e Historia. Sus investigaciones se han enfocado en temas de cultura regional, campesinado, ruralidades, globalización, sustentabilidad y patrimonio cultural en varios estados del país.

Rodolfo Humberto Aceves Arce es doctor en ciencias sociales con especialidad en antropología social por el Centro de Investigaciones y Estudios Superiores en Antropología Social (CIESAS), unidad Occidente. Es investigador asociado del proyecto del Fondo Mixto Jalisco- CIESAS Occidente, clave 2011-05-172363, y profesor en la licenciatura en antropología social en el Centro Universitario de Ciencias Sociales y Humanidades de la Universidad de Guadalajara y de los posgrados en Movilidad urbana, transporte y territorio en el Centro Universitario de Tonalá de dicha institución. Sus líneas de investigación son los estudios sociales urbanos, del deporte, la violencia colectiva y la cultura e identidades sociales.

José Alejos García es investigador titular del Centro de Estudios Mayas de la Universidad Nacional Autónoma de México (UNAM) y profesor de asignatura del Posgrado en Estudios Mesoamericanos de la misma casa de estudios. Es doctor en antropología lingüística por la UNAM en 1995. Sus investigaciones antropológicas se han centrado en los pueblos mayas de Chiapas y Guatemala, en temas de historia regional, identidad étnica, discurso narrativo y mitología, principalmente. Ahora desarrolla una teoría semiótica para el estudio de la literatura oral de los mayas contemporáneos.

\section{Traducción}

Jaime Sanromán Ruiz es egresado de la maestría en antropología social (2013) de la Universidad Iberoamericana, ciudad de México, donde es profesor de asignatura. Pertenece a la Asociación de Cronistas del Distrito Federal desde 2009. En 2007 publicó el artículo "Música tradicional e industria discográfica", en Antropología (núm. 80) y “Santiago Apóstol, ¿en México?”, en Peregrino (núm. 113, Logroño, España). “Diez mil tamales pa' mi santo, procesión nocturna” se encuentra en prensa dentro de la memoria del II Foro sobre Patrimonio Intangible (Inmaterial) de los Pueblos y Barrios Originarios en la Ciudad de México. En 2013 presentó el trabajo "Expresiones de religiosidad popular y relaciones sociales en Nextipac" en la tercera edición de dicho Foro y "Una mirada a la persona desde San Pedro Chiautzingo: 'ayuda', 'respeto' e 'interdependencia” en el Congreso Internacional Los pueblos indígenas de América Latina, siglos XIX-XXI.

\section{FotografíA}

Agradecemos profundamente a todos los que proporcionaron de manera generosa material gráfico para este número de Desacatos: Archivo de Manuel Basaldúa, Cecilia Dávalos Murillo por la imagen del Archivo Eusebio Dávila, Patricia de los Ríos, Ricardo Duarte Bajaña, Laura González, Carmen Icazuriaga a través de la Cátedra "Ángel Palerm", Roger Magazine, Marisol Pérez Lizaur, Arturo Pérez Rivera, Programa de Posgrado en Antropología de la Universidad Iberoamericana y Laboratorio Audiovisual del ciesAs. 